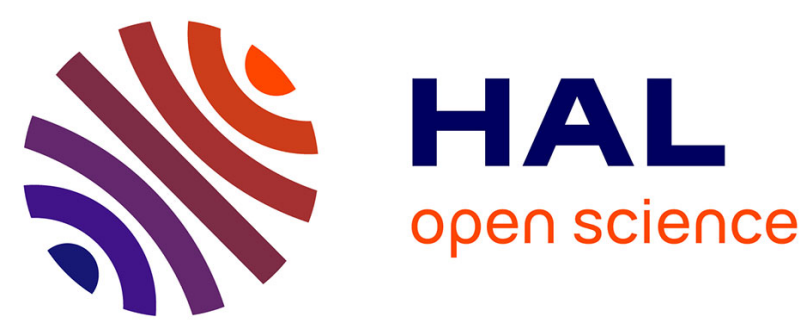

\title{
A comparison of converse magnetoelectric coupling effect of YIG film on FE and AFE ceramic substrates
}

Liuyang Han, Freddy Ponchel, Denis Remiens, Tuami Lasri, Nicolas Tiercelin, Genshui Wang, Philippe Pernod

\section{- To cite this version:}

Liuyang Han, Freddy Ponchel, Denis Remiens, Tuami Lasri, Nicolas Tiercelin, et al.. A comparison of converse magnetoelectric coupling effect of YIG film on FE and AFE ceramic substrates. Ferroelectrics, 2020, 557 (1), pp.1-8. 10.1080/00150193.2020.1713349 . hal-02963630

\section{HAL Id: hal-02963630 \\ https://hal.science/hal-02963630}

Submitted on 13 Oct 2020

HAL is a multi-disciplinary open access archive for the deposit and dissemination of scientific research documents, whether they are published or not. The documents may come from teaching and research institutions in France or abroad, or from public or private research centers.
L'archive ouverte pluridisciplinaire HAL, est destinée au dépôt et à la diffusion de documents scientifiques de niveau recherche, publiés ou non, émanant des établissements d'enseignement et de recherche français ou étrangers, des laboratoires publics ou privés. 


\title{
A comparison of converse magnetoelectric coupling effect of YIG film on FE and AFE ceramic substrates
}

\author{
Han Liuyang ${ }^{a}$, Ponchel Freddy ${ }^{a}$, Remiens! Denis ${ }^{a}$, Lasri Tuami ${ }^{b}$, Tiercelin Nicolas $^{c}$, Wang \\ Genshui ${ }^{d}$, and Pernod Philippe ${ }^{\mathrm{C}}$ \\ ${ }^{a}$ CNRS, UMR, IEMN-DOAE, Univ. Polytechnique Hauts-de-France, Valenciennes, France; \\ ${ }^{\mathrm{b}} \mathrm{CNRS}$, UMR, IEMN, Univ. Lille, Villeneuve d'Ascq, France; \\ ${ }^{c}$ Centrale Lille, ISEN, UMR, IEMN, Univ. Lille, Villeneuve d'Ascq, France; ${ }^{d}$ Shanghai Institute of Ceramics, Chinese Academy of \\ Science, Shanghai, China
}

\begin{abstract}
The magnetoelectric properties of laminated heterostructures consisting of ferromagnetic yttrium iron garnet (YIG) films deposited on antiferroelectric (AFE) ( $\left.\mathrm{Pb}_{0.97} \mathrm{La}_{0.02}\right)\left(\mathrm{Zr}_{0.6} \mathrm{Sn}_{0.3 \mathrm{Ti}} \mathrm{Ti}_{1}\right) \mathrm{O}_{3}$ (PLZST) and on ferroelectric ( $\mathrm{FE}) 0.75 \mathrm{~Pb}\left(\mathrm{Mg}_{1 / 3} \mathrm{Nb}_{2 / 3}\right) \mathrm{O}_{3}-0.25 \mathrm{~Pb}\left(\mathrm{Zr}_{0.48} \mathrm{Ti}_{0.52}\right) \mathrm{O}_{3}$ (PMN-PZT) ceramics are compared in this work. In particular, the Converse Magneto Electric (CME) coefficient is evaluated for these two types of structures. When the electric field is applied on an AFE ceramic, the CME coefficient peaks at the switching field and, without bias magnetic field, the maximum value is $11.6 \times 10^{8} \mathrm{~S} / \mathrm{m}$. In case of a FE ceramic, at the electric coercive field, the CME coefficient can reach $17 \times 10^{8} \mathrm{~S} / \mathrm{m}$ at 0 Oe. Each structure has advantages and dis-advantages depending on the applications.
\end{abstract}

\section{KEYWORDS}

Ferroelectric and antiferroelectric ceramics; magnetic film; multiferroic heterostructures; converse magneto electric coefficient

\section{Introduction}

Multiferroic materials with two or more ferroic properties have been largely studied these years and have offered many opportunities in developing novel functional devices [1-4]. The magnetoelectric (ME) materials, which display ferroelectricity and ferromagnetism simultaneously, can be obtained from single phase multiferroic compounds or artificial composites. The "artificial" ME composites have been extensively investigated by using ferroelectric materials and various heterostructures of ferroelectric (FE) and ferromagnetic (FM) materials. These kind of structures have found applications in different fields, such as magnetic sensors, magnetoelectric random access memories, volt-age tunable microwave devices like, resonators, filters, etc.[5-12].

Actually, the main interest has been focused on FM/FE heterostructures and in particular on the electric field tuning of magnetic response resulting from the strain of the FE component transferred to the FM component, the so-called converse magnetoelectric effect (CME). As AFE materials enjoy a large strain change during the electric-field induced AFE-FE transition, and display zero polarization and strain in a relaxed state $[13,14]$, there is a potential benefit for their use. The high electric-field induced phase transition strain makes that AFE-based ME composites are also interesting and can yield a good tuning of magnetic response with electric field. Another advantage is that the non-remnant state results in no pre-poling process which is a very important point from an application point of view [15]. Thus it is important to compare FM/FE (AFE) potentialities as a function of the desired properties. It is interesting to note that the electric-field control of magnetism in AFE-based composites has been rarely demonstrated [16]. In this paper we propose to compare the performances of FE and AFE ceramics coupled with a magnetic material in terms of CME. The magnetic material selected for the demonstration is yttrium iron garnet (YIG). This choice is mainly motivated by the applications targeted. Actually, thanks to its outstanding advantages including small magnetic anisotropy, narrow ferromagnetic resonance line width, high resistivity, and low microwave losses it is a good candidate for tunable multiferroic microwave devices. To understand the converse magnetoelectric (CME) coupling effect, it is necessary to study the electric-field tuning of magnetism of YIG at static state, which is seldom investigated. The FE and AFE materials chosen are $0.75 \mathrm{~Pb}\left(\mathrm{Mg}_{1 / 3} \mathrm{Nb}_{2 / 3}\right) \mathrm{O}_{3}-0.25 \mathrm{~Pb}\left(\mathrm{Zr}_{0.48} \mathrm{Ti}_{0.52}\right) \mathrm{O}_{3}$ (PMN-PZT) and $\left(\mathrm{Pb}_{0.97 \mathrm{La}} \mathrm{L}_{02}\right)\left(\mathrm{Zr}_{0.6} \mathrm{Sn}_{0.3} \mathrm{Ti}_{0.1}\right) \mathrm{O}_{3}$ (PLZST) respectively, these materials are fabricated at Shanghai Institute of Ceramics, Chinese Academy of Science. 


\section{Experimental}

The multiferroic composites are designed to be a layered structure of YIG film and PLZST or PMN-PZT ceramics with top and bottom electrodes in Pt. The ceramics were fabricated by the conventional solidstate reaction. Then the Pt electrodes $(100 \mathrm{~nm})$ were deposited on the both sides of the ceramics by magnetron sputtering. The YIG films were deposited on Pt/PLZST (PMN-PZT)/Pt structures by radiofrequency magnetron sputtering in a thickness of $400 \mathrm{~nm}$. The dimensions of the ceramic substrates and Pt electrodes are respectively $10 \times 10 \mathrm{~mm}^{2}$ and $6 \times 6 \mathrm{~mm}^{2}$, while the YIG film is $5 \times 3 \mathrm{~mm}^{2}$; metallic masks are used to size each of these areas. The PLZST and the PMN-PZT ceramic substrates are polycrystalline, they exhibit isotropic in-plane strain when the electric field is perpendicular to the plane. The YIG film being polycrystalline it demonstrates isotropic in-plane magnetic properties; hence, the magnetic field direction is parallel to the substrate plane. The schematic of YIG/Pt/PLZST (PMN-PZT)/Pt ME heterostructures and the way that electric and magnetic fields are applied are given in Figure 1.
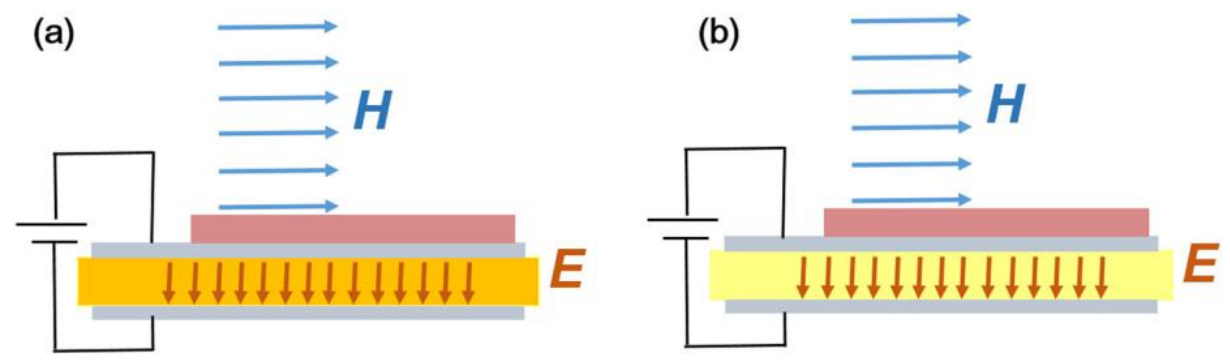

\section{YIG film Pt electrodes PLZST ceramic PMN-PZT ceramic}

Figure 1. (a) Schematic of the hetero structure YIG/Pt/PLZST/Pt and (b) schematic of the hetero structure YIG/Pt/PMN-PZT/Pt.
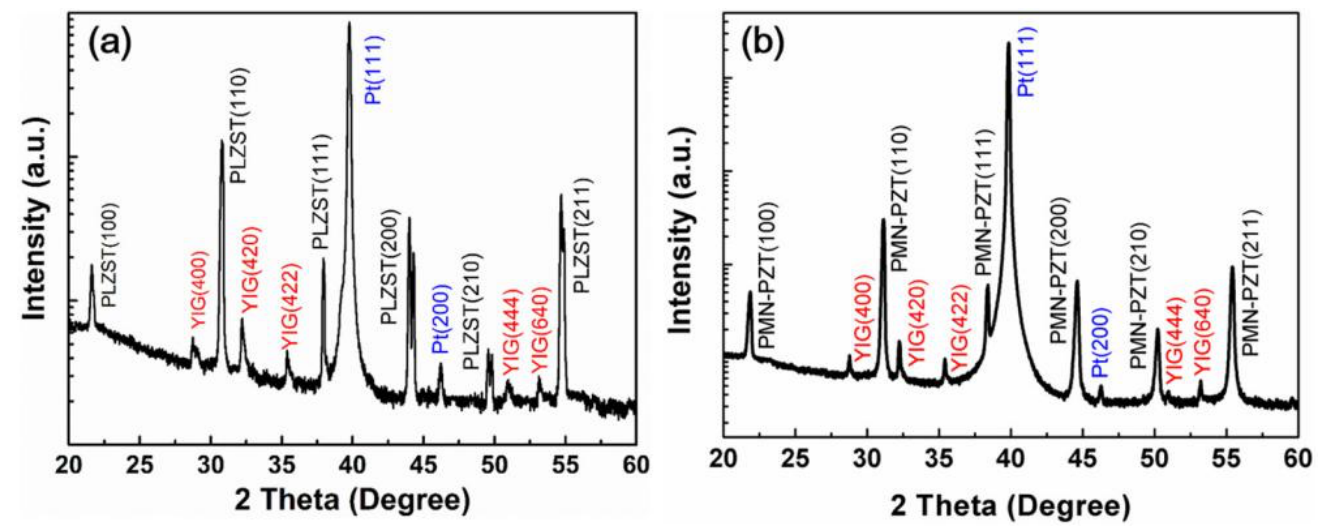

Figure 2. (a) X-rays diffraction of the hetero structure YIG/Pt/PLZST/Pt and (b) X-rays diffraction of the hetero structure YIG/Pt/PMN-PZT/Pt.

The X-ray diffraction (XRD) of the heterostructures was performed using a Rigaku $D$ x-ray diffractometer. Figure 2(a,b) are relative to the XRD of YIG/Pt/PLZST/Pt/and YIG/Pt/PMN-PZT/Pt respectively. These spectrums confirm the expected phase structures for all the materials. The origin of the various peaks is as labeled in the Figures. Even though the diffraction peaks of YIG thin films are of much lower intensity when compared to the peaks of the ceramic substrates and Pt electrodes, they are distinct enough and all correspond to pure YIG phase. The electric hysteresis loop (P-E) and fieldinduced strain curve (S-E) of the PMN-PZT/PLZST were obtained with a standard ferroelectric tester (TF analyzer 2000, aixACCT, Germany). The magnetic hysteresis loops (M-H) with different electric fields and the electric field tuning of magnetization (M-E) were measured at room temperature by a Vibration Sample Magnetometer (VSM, ADE model EV9) combined with an external DC voltage supply (Figure 3). It has to be noticed that all measurements are calibrated to offset the influence of the substrate, electrodes and wires. Thus the magnetic response of YIG film only is recorded. 


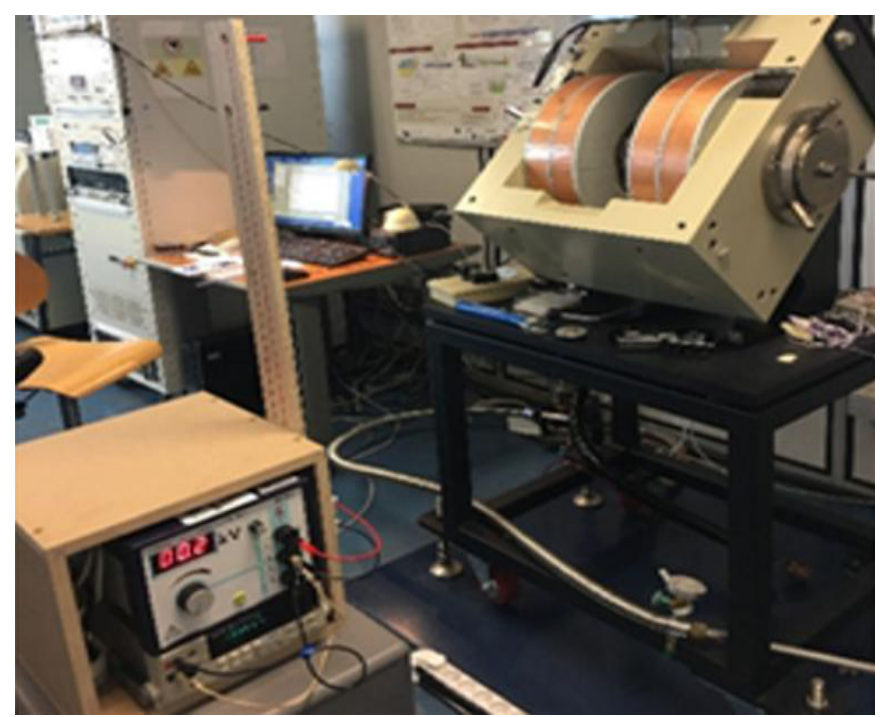

Figure 3. Photograph of the experimental setup.
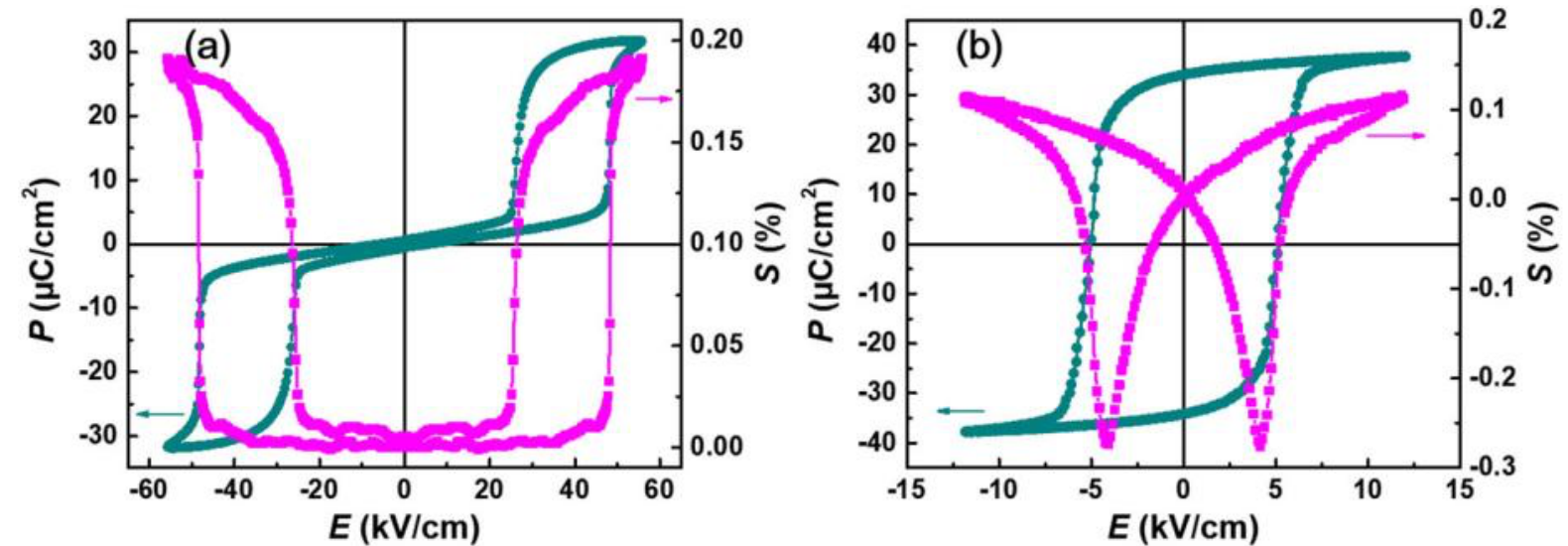

Figure 4. (a) P-E and S-E properties of the PLZST AFE ceramic and (b) P-E and S-E properties of the PMNPZT FE ceramic.

\section{Results and discussion}

Figures $4(a, b)$ show the electric hysteresis loops and electric-field-induced strain curves of PLZST and PMN-PZT ceramics respectively. The antiferroelectric characteristics of double P-E loops and horn-like S-E curves are clearly observed in PLZST (Figure 4(a)). The polarization and strain are nearly zero when the electric field is lower than the switching field. The zero-remnant state makes AFE materials free from the influence of electric load history and eliminates the pre-poling process that is necessary for FE materials. The switching fields of AFE-FE and FE-AFE phase transition are $50 \mathrm{kV} / \mathrm{cm}$ and 25 $\mathrm{kV} / \mathrm{cm}$ respectively, and the maximum strain can reach $0.2 \%$. The sharp strain change generated by the electric-field-induced structure phase transition gives us possibilities to obtain in-plane strain-mediated mag-netism transformation via the electric field application. In case of PMN-PZT (Figure 4(b)), the coercive field $\left(E_{C}\right)$ is $4.2 \mathrm{kV} / \mathrm{cm}$ and the butterfly S-E curve shows a very sharp strain peak at the electric coercive field. One can also note that the strain level rises from $-0.28 \%$ at $\mathrm{E}_{\mathrm{c}}$ to $+0.12 \%$ at $12 \mathrm{kV} / \mathrm{cm}$.

In the following the electric-field dependence of magnetic hysteresis (M-H) loops for both heterostructures (YIG/Pt/PMN-PZT/Pt and YIG/Pt/PLZST/Pt) is illustrated (Figure 5).

First, it is noticed that without application of an electric field the YIG film shows good magnetic properties on PMN-PZT ceramic substrate (Figure 5(a)). Since the PMN-PZT reaches the maximum negative strain at $E=E_{C}$ and the maximum positive strain at $E=E_{\max }$, the $(M-H)$ loops of $Y I G / P t / P M N-P Z T / P t$ at an electric field of $-4 \mathrm{kV} / \mathrm{cm}$ and $12 \mathrm{kV} / \mathrm{cm}$ are given as representatives. It can be seen that the positive strain tends to upright $(\mathrm{M}-\mathrm{H})$ loops, while the negative strain tends to slanted $(\mathrm{M}-\mathrm{H})$ loops, revealing the strain-mediated transformation of magneto crystalline anisotropy. It also reveals that the distinct E- 
induced magnetization variations occur at low magnetic fields (as zoomed in the inset [0 - $300 \mathrm{Oe}]$ (Figure 5(a)), while the E-induced magnetization variations are small at saturated magnetic field. In Figure 5(b), the remnant magnetization $\left(\mathrm{M}_{\mathrm{r}}\right)$ and magnetic coercive field $\left(\mathrm{H}_{\mathrm{c}}\right)$ variation as a function of electric field are given for YIG/Pt/PMN-PZT/ Pt. The sharp decrease of strain at E = $E_{c}$ results in a large jump in magnetization at an electric field of $-4 \mathrm{kV} / \mathrm{cm}$, and the $\mathrm{H}_{\mathrm{c}}$ shifts up to $29 \mathrm{Oe}$. Additionally, it is exhibited that the (Mr-E) and ( $\left.\mathrm{H}_{\mathrm{c}}-\mathrm{E}\right)$ curves of YIG/Pt/PMN-PZT/Pt heterostructure present a butterfly shape similar to the (S-E) curve of PMN-PZT ceramic, which is attributed to the correlation between strain and electric field.
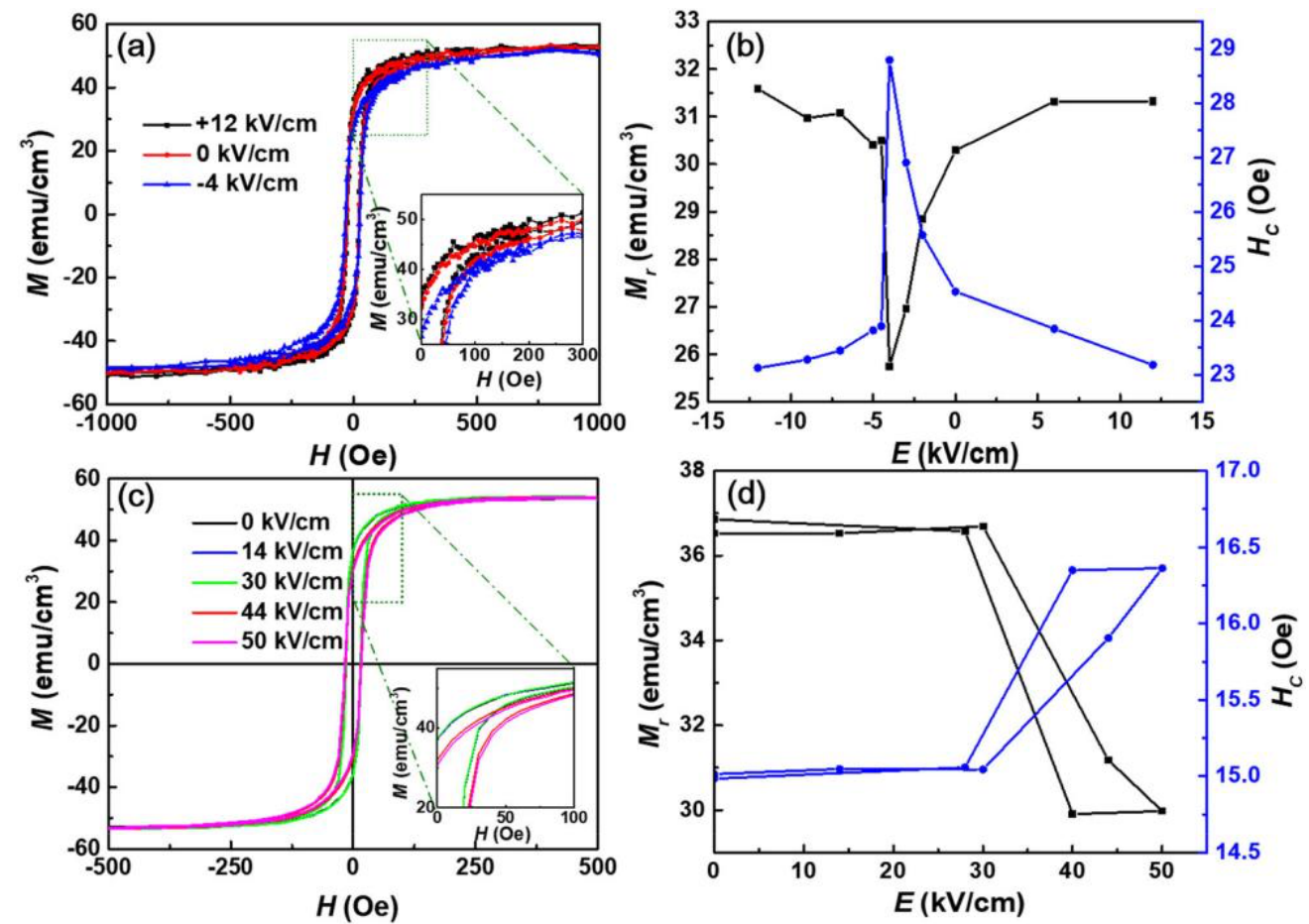

Figure 5. (a) M-H loops under different electric fields and (b) $M_{r}-E$ and $H_{c}-E$ curves of the hetero structure YIG/Pt/PMN-PZT/Pt, (c) M-H loops under different electric fields and (d) Mr-E and Hc-E curves of the hetero structure YIG/Pt/PLZST/Pt.

In order to compare the performances, exactly the same measurements have been performed with PLZST ceramic substrates. The electric-field dependence of magnetic hysteresis $(\mathrm{M}-\mathrm{H})$ loops of YIG/Pt/PLZST/Pt heterostructure is illustrated in Figure 5(c).

Without the application of an electric field, we observe that the YIG film shows the same performances as on PMN-PZT ceramic substrate. When the external electric field is lower than AFE-FE switching field of PLZST, the M-H loops match well with the one without electric field. Once the electric field is higher than AFE-FE switching field, the remnant magnetization decreases sharply, and the $\mathrm{M}-\mathrm{H}$ loops become difficult to be saturated. In this case, an induced magnetic coercive field shift is generated in response to the application of an electric field. The strain driven by the electric-field-induced AFE-FE phase transition is transferred through Pt electrodes to YIG film, which leads to magneto crystalline anisotropy transformation in the YIG film. In Figure 5(d), the remnant magnetization $\left(\mathrm{M}_{\mathrm{r}}\right)$ and magnetic coercive field $\left(\mathrm{H}_{\mathrm{c}}\right)$ variation as a function of positive applied electric field are given. The variations of $\mathrm{M}_{\mathrm{r}}(\mathrm{a}$ decrease of typically $20 \%$ ) and $\mathrm{H}_{c}$ (an increase of $10 \%$ ) appear at the electric-field-induced phase switching.

After all these characterizations, we have investigated the relative magnetization change $\left(\Delta M / M_{0}\right)$ for both cases (YIG/Pt/PMN-PZT/Pt and YIG/Pt/PLZST/Pt). The results obtained at 0 Oe are given in Figure $6(\mathrm{a}, \mathrm{c})$, respectively.

The butterfly-like shape of $\left(\Delta M_{M} / M_{0}-E\right)$ curve observed in Figure $6(a)$ is very similar to the S-E curve observed in PMN-PZT ceramic, which once again confirms that the change of magnetization in YIG film results from E-induced strain in the PMN-PZT ceramic. The positive strain favors magnetization process, while negative strain leads to demagnetization process. The large negative strain at $E_{c}$ facilitates a sharp reduction in magnetization, which yields a maximum of $\left(\Delta M / M_{0}\right)$ variations. Concerning PLZST ceramic, one can notice that the relative magnetization change $\left(\Delta M / M_{0}\right)$ generated in response to the application of an electric field results in a horn-like shape, quite similar to the one found for the strainfield response, suggesting a strong strain-driven magnetoelectric coupling between YIG and PLZST 
substrate. Finally, the determination of the two CME coefficients $\left(\alpha_{\mathrm{CME}}(\mathrm{E})\right)$, calculated from the equation: $\alpha_{\mathrm{CME}}=\mu_{0} \delta \mathrm{M} / \delta \mathrm{E}$, is performed (Figure 6(b,d)). It is noticed that the CME coefficient obtained from the YIG/Pt/PMN-PZT/Pt hetero-structure peaks at $E_{\mathrm{C}}$, reaching a large value of $17 \times 10^{8} \mathrm{~S} / \mathrm{m}\left(\alpha_{\mathrm{CME}}\right.$ in the absence of bias magnetic field) whereas the one for the YIG/Pt/PLZST/Pt heterostructure peaks at $11.6 \times 10^{8} \mathrm{~S} / \mathrm{m}$
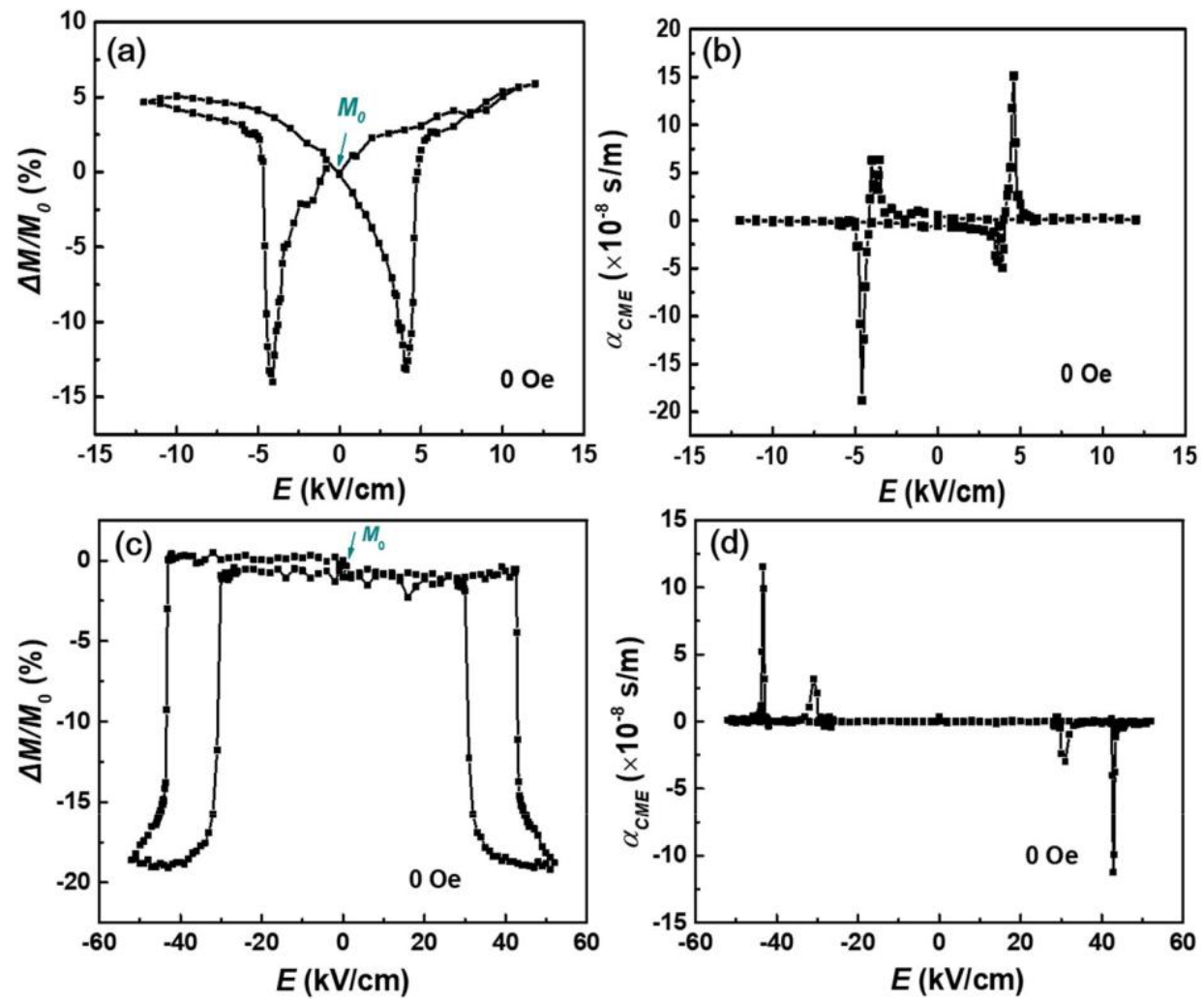

Figure 6. (a) $\Delta M / \mathrm{M}_{0}-E$ curve and (b) a CME-E curve of the hetero structure $Y I G / P t / P M N-P Z T / P t$, (c) $\Delta M / M_{0}-$ $\mathrm{E}$ curve and (d) $\alpha$ CME $-\mathrm{E}$ curve of the hetero structure $\mathrm{YIG} / \mathrm{Pt} / \mathrm{PLZST} / \mathrm{Pt}$.
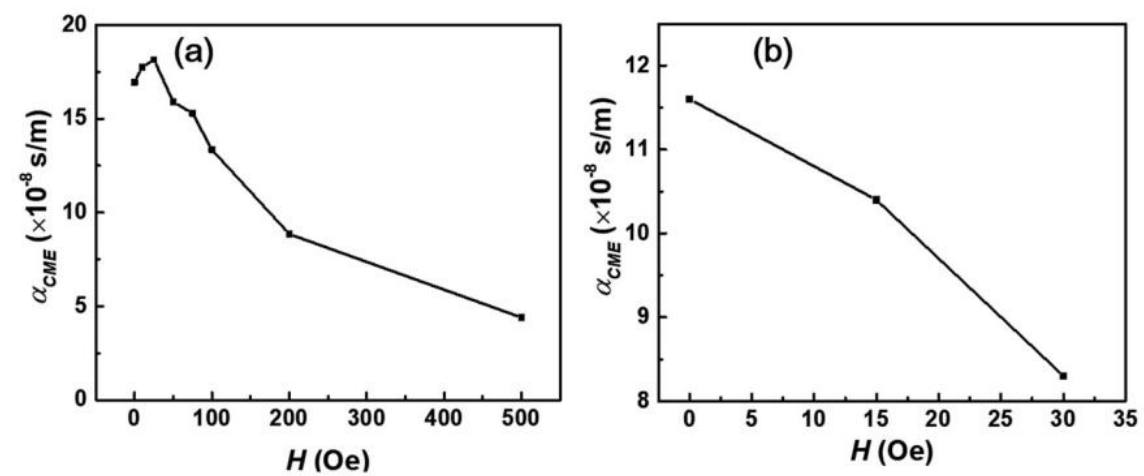

Figure 7. (a) The $\alpha_{\mathrm{CME}}-\mathrm{E}$ curve of the hetero structure YIG/Pt/PMN-PZT/Pt and (b) $\alpha_{\mathrm{CME}}-\mathrm{E}$ curve of the hetero structure YIG/Pt/PLZST/Pt.

To complete the study, we have determined the CME coefficient as a function of the bias magnetic field for both structures. The results are reported on Figure 7. One can note that first for a FE ceramic $\alpha_{\mathrm{CME}}$ rises and then decreases with the bias magnetic field. The maximum of $\alpha_{\mathrm{CME}}\left(18 \times 10^{8} \mathrm{~S} / \mathrm{m}\right)$ appears at $25 \mathrm{Oe}$, which can be attributed to the ease of switching the magnetization at this point because the $\mathrm{H}_{\mathrm{c}}$ is close to this value. When the bias magnetic field is higher than $\mathrm{H}_{\mathrm{c}}$, the electric-field-induced demagnetization process is hindered by large bias magnetic field that results in low CME coefficient. In case of the AFE ceramic the $\alpha_{\mathrm{CME}}$ for three different bias magnetic fields $(0,15,30 \mathrm{Oe})$ shows a continuous decrease. It is worth noting that the maximum $\alpha_{\mathrm{CME}}$ is obtained with a bias magnetic field of $0 \mathrm{Oe}$. The fact that the best performance of CME coupling is obtained without magnetic field is a really important asset in terms of design when targeting ME devices conception. The maximum $\alpha$ CME value recorded is $11.6 \times 10^{8} \mathrm{~S} / \mathrm{m}$. 
In fact, this study points out that the ME coefficients obtained for FE and AFE ceramics exhibit about the same peak values. So, from an application point of view this coefficient does not appear as a discriminating factor. The choice of the structure will be more conditioned by the operating conditions (electric and magnetic fields level).

\section{Conclusion}

In summary, the E-field controlled magnetism in AFE/FE -based multiferroic hetero-structures with the same magnetic material: YIG in thin form is compared for the first time, to the best of the authors' knowledge. In both cases, the electric-field-dependent (M-H) loops curves reveal the strain-mediated transformation of magnetic anisotropy in YIG film. The CME coefficient can reach $17 \times 10^{8} \mathrm{~S} / \mathrm{m}$ in the absence of bias magnetic field at the Ec of PMN-PZT ceramic, and the maximum of CME coefficient $\left(18 \times 10^{8} \mathrm{~S} / \mathrm{m}\right)$ is observed under the magnetic and electric fields of 25 Oe and $4.2 \mathrm{kV} / \mathrm{cm}$ respectively. For AFE, the maximum $\alpha_{\mathrm{CME}}$ is $11.6 \times 10^{8} \mathrm{~S} / \mathrm{m}$ without magnetic field. As a result, we have demonstrated that it is possible to obtain a good control of the magnetization of the YIG film through the strains induced by the electric field and a strong converse magnetoelectric effect with FE or AFE materials. It is noticed that the maximum values of the CME coefficients are in the same order. This is due to the fact that the strain levels for AFE or FE ceramics used in these experiments are very similar. The AFE material presents the advantage of its unique zero-remnant state which gives us the possibility to avoid prepoling process but a large voltage is necessary to observe the coupling effect. Thus, the implementation of this type of structure from an application point of view requires reducing the phase switching induced by the electric field while maintaining the stress level. This compromise can be difficult to achieve. Concerning the heterostructure with a FE material, the lower control voltages needed are an asset in the context of a MMIC circuits.

\section{References}

[1] W. Eerenstein, N. D. Mathur, and J. F. Scott, Multiferroic and magnetoelectric materials, Nature. 442 (7104), 759 (2006). DOI: 10.1038/nature05023.

[2] Y. Wang et al., Multiferroic magnetoelectric composite nanostructures, NPG Asia Mater. 2 (2), 61 (2010). DOI: 10.1038/asiamat.2010.32.

[3] C. W. Nan et al., Multiferroic magnetoelectric composites: historical perspective, status, and future directions, J. Appl Phys. 103 (3), 031101 (2008). DOI: 10.1063/1.2836410.

[4] J. M. Hu et al., Multiferroic magnetoelectric nanostructures for novel device applications, MRS Bull. 40 (9), 728 (2015). DOI: 10.1557/mrs.2015.195.

[5] G. Srinivasan, Magnetoelectric composites, Annu. Rev. Mater. Res. 40 (1), 153 (2010). DOI: 10.1146/annurev-matsci-070909-104459.

[6] J. Zhai et al., Magnetoelectric laminate composites: an overview, J. Am. Ceramic Soc. 91 (2), 351 (2008). DOI: 10.1111/j.1551-2916.2008.02259.x.

[7] T. X. Nan et al., Voltage impulse induced bistable magnetization switching in multiferroic heterostructures, Appl. Phys. Lett. 100 (13), 132409 (2012). DOI: 10.1063/1.3698363.

[8] A. S. Tatarenko, G. Srinivasan, and M. I. Bichurin, Magnetoelectric microwave phase shifter, Appl. Phys. Lett. 88 (18), 183507 (2006). DOI: 10.1063/1.2198111.

[9] X. Yang et al., Voltage tunable multiferroic phase shifter with YIG/PMN-PT heterostruc-ture, IEEE Microw. Wireless Compon. Lett. 24 (3), 191 (2014). DOI: 10.1109/LMWC.2013. 2292924.

[10] A. B. Ustinov, G. Srinivasan, and Y. K. Fetisov, Microwave resonators based on single-crystal yttrium iron garnet and lead magnesium niobate-lead titanate layered structures, J Appl Phys. 103 (6), 063901 (2008). DOI: $10.1063 / 1.2841200$.

[11] Y. K. Fetisov, and G. Srinivasan, Electric field tuning characteristics of a ferrite-piezoelec-tric microwave resonator, Appl. Phys. Lett. 88 (14), 143503 (2006). DOI: 10.1063/1. 2191950.

[12] A. S. Tatarenko et al., Microwave magnetoelectric effects in ferrite-piezoelectric compo-sites and dual electric and magnetic field tunable filters, J. Electroceram. 24 (1), 5 (2010). DOI: 10.1007/s10832-0079382-1.

[13] S. Lepadatu and M. Vopson, Heat-assisted multiferroic solid-state memory, Materials. 10 (9), 991 (2017). DOI: 10.3390/ma10090991.

[14] X. Hao et al., A comprehensive review on the progress of lead zirconate-based antiferro-electric materials, Prog Mater Sci. 63, 1 (2014). DOI: 10.1016/j.pmatsci.2014.01.002.

[15] Z. Zhou et al., Antiferroelectric materials, applications and recent progress on multiferroic heterostructures, SPIN. 05 (01), 1530001 (2015). DOI: 10.1142/S2010324715300017.

[16] Z. Zhou et al., Strong non-volatile voltage control of magnetism in magnetic/antiferroelec-tric magnetoelectric heterostructures, Appl. Phys. Lett. 104 (1), 012905 (2014). DOI: 10. 1063/1.4861462. 